\title{
nature
}

\section{Biology versus physics?}

There is a tendency for biology to dominate perceptions of science at the expense of support for other disciplines. There is no scientific justification for that situation, which also carries economic dangers.

$\mathrm{P}$ aradigm is a problematic word. Thanks to the late Thomas Kuhn, who commandeered it for his own philosophical purposes, it implicitly carries with it the notion that only physicists these days can experience true scientific revolutions. A Kuhnian revolution involves the acceptance of a new conceptual framework for scientific models that is incommensurate with (or cannot be derived by extension of) the traditional framework. The older paradigm turns out to have produced predictions that are approximations of those, closer to observation, that are delivered by its successor.

Whereas physicists can point to Planck and Einstein as classic suppliers of new paradigms, there has always been difficulty in identifying biological conceptual frameworks that fit this definition. Darwin provided a framework where, pace various deities, there was little before. The elucidation of the structure ofDNA and of the genetic code started a revolution worthy of the title, but those were not incommensurate with any other ideas then accepted in chemistry or biology. The subsequent discovery of non-coding sequences within genes took everybody by surprise but was readily accommodated. Some have proposed Peter Mitchell's chemiosmotic theory (Nature 191, 144; 1961) as a Kuhnian revolutionary achievement (BioEssays 19, 93; 1996).

Biologists are not alone in occasionally using the word "paradigm" in a narrow context that arch-Kuhnians should thoroughly disapprove of - an improper substitute for "scenario" or, even more specifically, "model". (An example is the hypothesis, described by some as a paradigm, that particular molecules - neurotrophins - are the key to the competition between neurons for survival and to the formation of appropriate interneuron connections in the developing brain.)

On the other hand, non-Kuhnian revolutions in biology are often, even usually, sparked by innovations in technique. Some are biological developments - monoclonal antibodies, the polymerase chain reaction - while many other transformational tools have emerged from physics: X-rays, radioactivity, neutrons, positrons, nuclear magnetism, various microscopies, optical tweezers....

Forget about philosophy - that bio-relevance, say some physicists, is a reason to fund physics. How are the mighty fallen, others might respond. From their dominant positions at the heart of the science-industrial-government corpus during the middle decades of this century, physicists are now reduced to justifying their continuing existence on the coat-tails of another discipline.

It is one thing to argue, as this publication has, that disciplines need to look to themselves to produce effective advocacy. It is another to leap onto a superficial notion that any discipline has little more to offer. That dismissive tone comes from the very top, in the recent assertion of President Bill Clinton to the effect that the past 50 years have been the age of physics, whereas the next will be "very likely characterized predominantly as the age of biology". Supporters of the life sciences - some of them scientists who should know better quote that all too gleefully and glibly.

Physicists and chemists cannot take all the blame for the low public and political appreciation of their disciplines. Who can easily compete with the weekly announcements, trumpeted by the media worldwide, of a new genetic link, a new virus, or another step in the understanding of neural degeneration? And in the eyes of those who look more closely at the science, there is an appealing grandeur in the unfolding picture of underlying commonality, at the genetic and sub-cellular levels, between the kingdoms of life, let alone species.

Yet take, as one example of contemporary physics in particular, a recent paper that for the first time demonstrated quantum teleportation experimentally (Nature 390, 575; 1997). More media-friendly than most "small" physics ("teleportation" was leapt on in many newspaper reports), the result and the underlying principles are nevertheless difficult to comprehend by any standard. Yet for its technical quality, for its intrinsic scientific value within the quantum paradigm that underlies our understanding of light and matter, for its relevance to philosophical paradoxes associated with quantum mechanics, and for its technological potential, such a result, and others that will no doubt follow, gives much reason to celebrate and support the discipline that produced it.

\section{Dangers}

Some biologists, benefiting from politicians' justified appeals for increases in health research budgets (see for example page 114), protest that their discipline is not competing with others - as did the chief executive of the US Federation of American Societies for Experimental Biology, saying last week in the Washington Post that increases for health research are not intended to be at the expense of other sciences, which are funded from different budget lines. Given the pressures on public funds and the tone set by Clinton, that statement is questionable if not disingenuous.

Despite the dangers of its misrepresentation, Clinton's statement is appropriate as a celebration of biology's increasing relevance to countries' health and wealth. Meanwhile, physicists are rightly attracted by new applications of their work — to probing the structures, properties, dynamics and energetics of complex biomolecules, to non-invasive imaging, and to the analysis and simulation of biological networks and systems.

But, in the United States and in Europe, there is a growing danger that, as the attractiveness and impact of biology develops, an inadequate appreciation of physics will effectively lead to a backlash against it. Perhaps the only allies that physicists have are, for big science, the cultural appeal to the public of fundamental laws of nature and of the Universe. For small physics, as for non-biological chemistry, the principal allies are in industry.

Despite the end of the Cold War, defence and civil industrial interests in "small" science are as strong as ever. Yet attempts to marry industrial and scientific perspectives — such as the technology foresight exercises now carried out in several countries - fail to generate political resonance. Nevertheless, new chemicals, advanced materials, telecommunications, semiconductors and other electronic, magnetic and photonic technologies have major industrial relevance, as much significance for national economies as ever and an increasing impact on society. The fact that the results of physics in particular have a way of unobtrusively and unpredictably invigorating apparently unrelated disciplines and technologies, even to a revolutionary extent, is an additional benefit. As skill shortages grow, should not all that be something for politicians to worry more about? 